ARTICLE

\title{
Lithium-coated polymeric matrix as a minimum volume-change and dendrite-free lithium metal anode
}

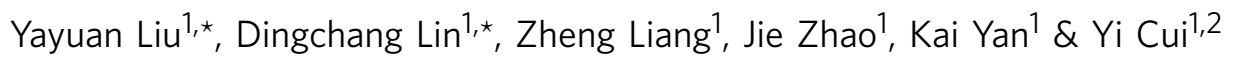

Lithium metal is the ideal anode for the next generation of high-energy-density batteries. Nevertheless, dendrite growth, side reactions and infinite relative volume change have prevented it from practical applications. Here, we demonstrate a promising metallic lithium anode design by infusing molten lithium into a polymeric matrix. The electrospun polyimide employed is stable against highly reactive molten lithium and, via a conformal layer of zinc oxide coating to render the surface lithiophilic, molten lithium can be drawn into the matrix, affording a nano-porous lithium electrode. Importantly, the polymeric backbone enables uniform lithium stripping/plating, which successfully confines lithium within the matrix, realizing minimum volume change and effective dendrite suppression. The porous electrode reduces the effective current density; thus, flat voltage profiles and stable cycling of more than 100 cycles is achieved even at a high current density of $5 \mathrm{~mA} \mathrm{~cm}^{-2}$ in both carbonate and ether electrolyte. The advantages of the porous, polymeric matrix provide important insights into the design principles of lithium metal anodes.

\footnotetext{
${ }^{1}$ Department of Materials Science and Engineering, Stanford University, Stanford, California 94305, USA. ${ }^{2}$ Stanford Institute for Materials and Energy Sciences, SLAC National Accelerator Laboratory, 2575 Sand Hill Road, Menlo Park, California 94025, USA. * These authors contributed equally to this work. Correspondence and requests for materials should be addressed to Y.C. (email: yicui@stanford.edu).
} 
T he ever-increasing demand for high-energy-density storage systems for transportation (electric vehicles), portable electronics and other applications has stimulated intensive research on rechargeable batteries that go beyond the conventional lithium (Li) ion chemistry ${ }^{1}$. Among all the possible options $^{2,3}, \mathrm{Li}$ metal is the most ideal anode material due to its high theoretical capacity $\left(3,860 \mathrm{mAhg}^{-1}\right)$ as well as its low electrochemical potential $(-3.040 \mathrm{~V}$ versus standard hydrogen electrode $)^{4,5}$. Despite the appealing properties, Li metal electrode has been plagued for decades with the problem of ramified growth during repeated stripping/plating and the associated electrolyte decomposition, which lead to serious safety concerns and poor battery cycling efficiency 6,7 .

It is well known that Li is highly reactive such that in liquid electrolyte it reacts spontaneously with solvent molecules and salt anions to form an insoluble layer of solid-electrolyte interphase $(\mathrm{SEI})^{8}$. When SEI becomes stabilized to block electron transfer, this passivating film can slow down or, ideally, prevent the electrolyte from further decomposition. Nevertheless, as a 'hostless' electrode, the Li metal anode has a virtually infinite relative volume change during stripping/plating, resulting in the mechanical instability of the SEI layer and the formation of cracks. The cracks expose fresh $\mathrm{Li}$ underneath and locally enhance the $\mathrm{Li}$ ion flux, leading to non-homogeneous Li growth (dendrite, filament, etc.) that can induce internal short circuit and thermal runaway with potential safety hazards ${ }^{9,10}$. Moreover, the large-surface-area, dendritic Li growth brings about a continuous loss of both working Li and electrolyte (recurrent SEI formation), which gives rise to low Coulombic efficiency (CE) and rapid capacity decay.

For the past four decades, continuous research on Li metal stripping/plating has deepened our understanding of the process, but has not helped in solving the above-mentioned problems in an effective manner ${ }^{11-14}$. On one hand, the use of solid electrolytes to suppress dendrite propagation remains premature in the current stage ${ }^{15-17}$, for they often fall short of meeting the high-power requirement at ambient temperature due to limited ionic conductivity ${ }^{18,19}$, together with issues such as large interfacial impedance $^{20,21}$. On the other hand, the most common approaches to dendrite mitigation in liquid electrolyte focus on the stabilization of SEI via adjusting the electrolyte composition and additives ${ }^{22-26}$. Though proven to be effective, most additives will be continuously consumed during battery cycling so that the suppression effect is not fully sustainable ${ }^{27-29}$. Alternatively, the application of a mechanically stable artificial SEI coating such as polymer or solid-state blocking layers has been proposed ${ }^{30-33}$. For example, a promising nanoscale interfacial engineering approach has been demonstrated recently based on interconnected hollow carbon nanospheres, ultrathin two-dimensional boron nitride or oxidized polyacrylonitrile fibres to control the dendrite growth and improve the cycling $\mathrm{CE}^{34-36}$. Nevertheless, all these studies adopted the galvanostatic $\mathrm{Li}$ plating/stripping approach on a current collector, which is still unable to address the issue of infinite volume change since the electrodes expand during Li plating and shrink during stripping. In addition, contrary to Li-ion batteries where Li ions are stored in prelithiated cathodes, many of the intensively studied high-energy-density battery chemistries (e.g., Li-air and Li-S) involve cathodes in the nonlithiated form. Therefore, it is apparent that a metallic $\mathrm{Li}$ anode design with no volume change at the whole-electrode scale and long-term cycling stability in liquid electrolyte is of paramount research importance ${ }^{3}$.

Herein, we demonstrate a rational design of metallic Li anode that successfully achieves minimum volume change at the wholeelectrode level and stable, dendrite-free $\mathrm{Li}$ cycling. Several important design principles are employed. Firstly, in order to realize negligible volume change, a chemically as well as electrochemically stable matrix is required to sustain a constant electrode volume during cycling. In addition, complete confinement of Li within the matrix is necessary to preserve a constant electrode dimension; therefore, the direct nucleation of $\mathrm{Li}$ on the top surface of the matrix should be prevented, as reported in previous studies ${ }^{31,34}$. Moreover, a porous electrode is desirable since a reduced local current density is beneficial to alleviate dendrite propagation ${ }^{37,38}$. Following the aforementioned rationale, a Li-coated polyimide (PI) matrix design for metallic $\mathrm{Li}$ anode is proposed. The electrospun polymeric fibres guarantee a chemically and electrochemically inert matrix, which is favourable to confine the stripping/plating of Li solely within the matrix. Notably, the choice of PI is rather unique for it is one of the only few high-performance polymers that exhibit excellent chemical stability, heat resistance and mechanical strength above the melting point of $\mathrm{Li}\left(180^{\circ} \mathrm{C}\right)^{39}$. However, the wetting of molten Li on PI polymer is poor. By applying a layer of $\mathrm{ZnO}$ coating via atomic layer deposition (ALD) on the PI fibres, we discover that molten $\mathrm{Li}$ can react with $\mathrm{ZnO}$ and subsequently infuse into the PI matrix, resulting in a free-standing, current collector-free Li electrode. More importantly, we separate the conducting function from the matrix, where infused metallic $\mathrm{Li}$ itself serves as the only electron transport media. As a result, the electrically insulating surface after Li stripping effectively prevents the direct plating of Li on the top surface of the matrix in the subsequent cycle, bringing about a well-confined, dendrite-free $\mathrm{Li}$ stripping/plating behaviour that successfully addresses the problem of infinite volume change present in all the previous designs. Moreover, the obtained electrode is highly porous so that the reduced effective current density results in flat voltage profiles and stable cycling of at least 100 cycles in both carbonate- and ether-based electrolytes even at a high current density of $5 \mathrm{~mA} \mathrm{~cm}^{-2}$, which stands in stark contrast to the fluctuated and unstable cycling profile of bare Li foil electrodes.

\section{Results}

Fabrication of the Li-coated PI electrode. Figure 1 illustrates the fabrication process of the Li-coated PI matrix electrode. We employed a facile electrospinning method to obtain the PI fibre matrix. Thermogravimetric analysis (Supplementary Fig. 1) confirmed that the electrospun PI fibre is stable up to $450^{\circ} \mathrm{C}$, which is well above the melting point of $\mathrm{Li}\left(180^{\circ} \mathrm{C}\right)$. Such high heat resistance ensures that the matrix can withstand the temperature of molten $\mathrm{Li}$ in order to fabricate the metallic $\mathrm{Li}$ anode. Nevertheless, molten Li cannot wet the bare PI matrix (Supplementary Fig. 2). Due to the high surface tension of molten Li on PI fibre, a large driving force is needed for Li to infuse into the matrix. Rather than physical absorption, a surface chemical reaction that can afford much higher driving force is necessary. Through universal screening on materials that can undergo conversion reaction with $\mathrm{Li}$, we found that a layer of conformal $\mathrm{ZnO}$ coating applied to the matrix via ALD can render the matrix wet by molten $\mathrm{Li}$, or 'lithiophilic'. Subsequently, when the core-shell PI-ZnO matrix was put into contact with molten $\mathrm{Li}, \mathrm{ZnO}$ reacted with molten $\mathrm{Li}$ and, interestingly, extra Li can be drawn into the matrix, affording a Li-coated PI electrode.

Characterization of the Li-coated PI electrode. The morphology of the electrospun PI fibres was characterized using scanning electron microscopy (SEM). As can be seen from Fig. 2a, the fibres were continuous and uniform in general with a diameter of $\sim 400 \mathrm{~nm}$. After ALD coating, the surface of the fibres roughened due to the accumulation of a conformal layer of 


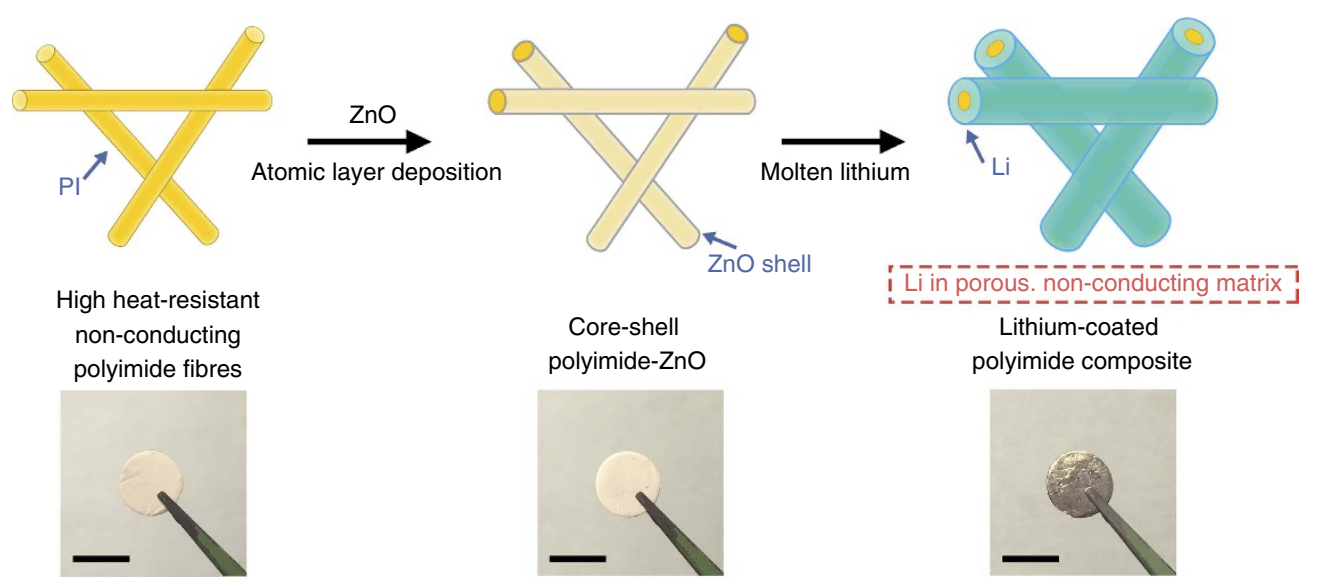

Figure 1 | Schematic of the fabrication of the Li-coated PI matrix. Electrospun PI was coated with a layer of ZnO via ALD to form core-shell PI-ZnO. The existence of $\mathrm{ZnO}$ coating renders the matrix 'lithiophilic' such that molten $\mathrm{Li}$ can steadily infuse into the matrix. The final structure of the electrode is $\mathrm{Li}$ coated onto a porous, non-conducting polymeric matrix. Scale bar, $1 \mathrm{~cm}$.

$\mathrm{ZnO}$ nanoparticles (Fig. 2b). Evident PI-ZnO core-shell structure can be observed from the cross-sectional SEM image (Fig. 2c), where the PI core appeared darker in colour and the thickness of the $\mathrm{ZnO}$ shell was measured to be $\sim 30 \mathrm{~nm}$ (Supplementary Fig. 3). Scanning transmission electron microscopy energydispersive X-ray elemental mapping (Fig. 2d) as well as line scan (Supplementary Fig. 4) resolved the distribution of C (from the PI backbone) and $\mathrm{Zn}$ (from the $\mathrm{ZnO}$ coating), further confirming the core-shell structure of the fibre matrix after ALD. Figure $2 \mathrm{e}$ shows the SEM top view of the PI matrix after Li coating. It appears that $\mathrm{Li}$ was drawn into the matrix preferentially along the fibres, and hence the matrix was not densely coated with Li. The porous nature of the resulting matrix can be further revealed from the cross-sectional SEM image (Supplementary Fig. 5), where obvious pores can be observed. X-ray diffraction (XRD) was employed to understand the compositional evolution of the matrix (Fig. 2f). As can be seen clearly from the XRD spectra, the $\mathrm{ZnO}$ layer reacted with molten $\mathrm{Li}$ to form $\mathrm{LiZn}$ alloy and $\mathrm{Li}_{2} \mathrm{O}$ during the $\mathrm{Li}$ coating process ${ }^{40}$. Since the electron percolation pathway of the alloy particles within the non-conducting $\mathrm{Li}_{2} \mathrm{O}$ matrix is generally limited it is justified to believe that the matrix remained low in electrical conductivity compared to metallic Li after $\mathrm{Li}$ coating ${ }^{41,42}$. Such a porous and electrically insulating matrix renders favourable electrochemical features to the resulting $\mathrm{Li}$ electrode, which will be elaborated in detail in later sections.

It is important to ensure the stability of the polymeric backbone in contact with the highly reductive molten Li. Therefore, Fourier transform infrared spectroscopy was employed (Fig. 2g), where the existence of PI can be identified from three characteristic peaks corresponding to asymmetric $\mathrm{C}=\mathrm{O}$ stretching, symmetric $\mathrm{C}=\mathrm{O}$ stretching and $\mathrm{C}-\mathrm{N}$ stretching ${ }^{43}$. After ALD and the subsequent contact with molten $\mathrm{Li}$, the transmittance intensity was reduced due to the coating layers, while the three characteristic peaks remained, confirming the intact polymeric matrix. In addition, neither anodic nor cathodic decomposition peaks can be observed from the cyclic voltammetry of the pristine PI (Supplementary Fig. 6), indicating the stability of the polymeric matrix towards electrochemical cycling.

The capacity of the Li electrode was determined to be above $2,000 \mathrm{mAhg}^{-1}$ based on the weight of the whole composite electrode via Li stripping (Supplementary Fig. 7). Thus, the existence of the matrix did not seriously compromise the high specific capacity of the Li anode. Noticeably, by adjusting the thickness of the electrospun matrix, which can be done easily by changing the electrospinning time, the thickness of the final $\mathrm{Li}$ electrode can be tuned accordingly with great ease to match the capacity of the battery cathode (Supplementary Fig. 8), manifesting the facileness of our proposed method for real applications.

Well-confined Li stripping/plating within the matrix. We investigated the stripping/plating process of the Li-coated PI matrix using a two-electrode symmetric cell configuration assembled in 2,032 coin cells with carbonate-based electrolyte $\left(1 \mathrm{M}\right.$ lithium hexafluorophosphate $\left(\mathrm{LiPF}_{6}\right)$ in $1: 1$ ethylene carbonate (EC)/diethyl carbonate (DEC), BASF). Interestingly, the electrode exhibited a well-confined stripping/plating behaviour (Fig. 3). Top fibres of the matrix were exposed after stripping away $5 \mathrm{mAh} \mathrm{cm}^{-2} \mathrm{Li}$ at a current density of $1 \mathrm{~mA} \mathrm{~cm}^{-2}$ (Fig. 3a), which indicates that the top Li layers were dissolved more favourably during stripping. Subsequently, when $3 \mathrm{mAh} \mathrm{cm}{ }^{-2} \mathrm{Li}$ was plated, $\mathrm{Li}$ was observed to be deposited into the matrix and partially fill the space between the fibres (Fig. 3b). Finally, when all the stripped Li was plated back (Fig. 3c), the top surface of the matrix was covered again by Li (similar to Fig. 2e) with no discernable dendrites. The well-confined plating behaviour can be rationalized by the removal of the conductive $\mathrm{Li}$ component in the prior stripping process, and thus the exposure of the electrically insulating PI surface. Since Li plating only occurs where electrons meet Li ions, the exposed insulating surface was rendered unfavourable for $\mathrm{Li}$ nucleation. Instead, the metallic Li confined within the matrix served as the only electron conductor such that the deposition of Li occurred majorly on the underlying reserved Li. In addition, the much larger effective surface area (Supplementary Fig. 9) lowered the overall deposition barrier, thus preventing the formation of 'hot spot'. As a result, uneven Li deposition can be suppressed. On the contrary, if the electrons could be efficiently transported to the electrolyte-facing top surface or the electrodes exhibited limited surface area, undesirable Li stripping/plating behaviour may occur after recurrent cycles (Fig. 3d), as discussed in previous studies ${ }^{31,34}$. Direct Li nucleation on the top surface might be easier due to the high availability of both electrons and $\mathrm{Li}$ ions, which provides favourable sites for dendrite growth while leaving the interior voids empty.

Dendrite-free cycling with minimum volume change. The morphology of the top surface of the Li-coated PI matrix was studied after 10 cycles of galvanostatic stripping/plating in 

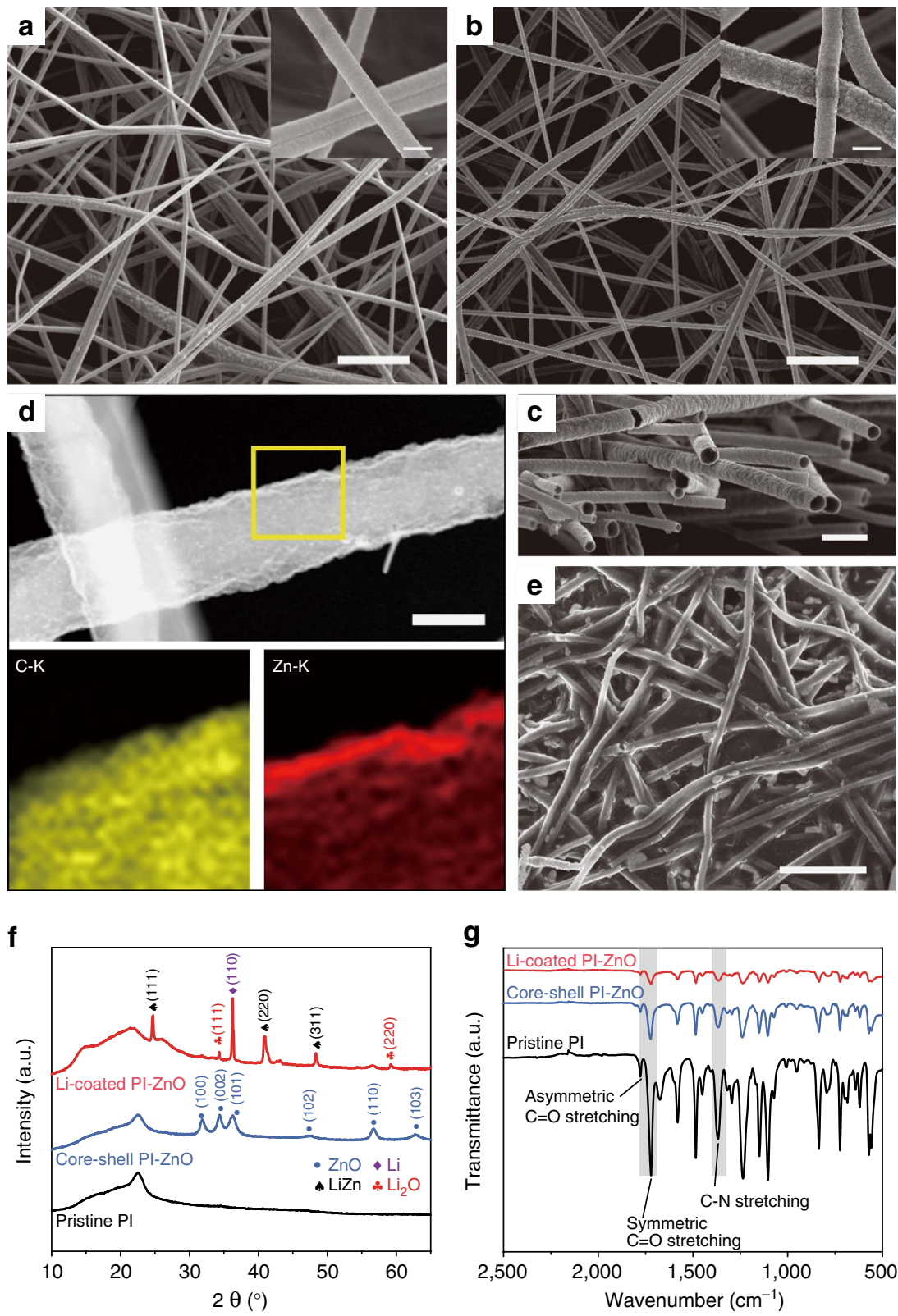

Figure 2 | Characterization of the Li-coated PI electrode. SEM images of the electrospun PI fibres (a) before and (b) after ZnO coating. Scale bars, $5 \mu$ m; insets scale bars, $500 \mathrm{~nm}$. (c) Cross-sectional SEM image of the core-shell PI-ZnO, where the conformal ZnO coating can be observed clearly from the contrast of the fibre cross-sections. Scale bar, $1 \mu \mathrm{m}$. (d) Scanning transmission electron microscopy (STEM) image of a single core-shell PI-ZnO fibre and the corresponding energy-dispersive X-ray (EDX) elemental mapping of C and Zn distribution. Scale bar, $200 \mathrm{~nm}$. (e) SEM image of the Li-coated PI matrix, showing the porous nature of the Li electrode. Scale bar, $5 \mu \mathrm{m}$. (f) XRD spectra of the pristine $\mathrm{Pl}$, the core-shell $\mathrm{PI}-\mathrm{ZnO}$ and the $\mathrm{Li}$-coated $\mathrm{PI}-\mathrm{ZnO}$ matrix, where the Li-coated PI-ZnO exhibited the signals of LiZn alloy, $\mathrm{Li}_{2} \mathrm{O}$ and metallic Li. (g) Fourier transform infrared spectra of the pristine PI, the core-shell $\mathrm{PI}-\mathrm{ZnO}$ and the Li-coated PI-ZnO matrix (Li was scraped away to expose the underlying matrix in order to obtain the signal). The characteristic peaks of PI remained after $\mathrm{ZnO}$ and $\mathrm{Li}$ coating, indicating the stability of the polymeric matrix.

EC/DEC (Fig. 4a-c, Supplementary Fig. 10). Due to the abovementioned well-confined Li cycling behaviour, the surface of the Li-coated PI electrode remained consistently flat even at a high current density of $5 \mathrm{~mA} \mathrm{~cm}^{-2}$ (note that the uniform fibrous features in Fig. $4 \mathrm{a}-\mathrm{c}$ are the fibre matrix and shall not be mistaken as $\mathrm{Li}$ dendrites). Moreover, no excessive dendrite formation can be observed after long-term cycling of 100 cycles (Supplementary Fig. 11). On the contrary, for the bare $\mathrm{Li}$ electrodes, rough surface and excessive mossy Li growth can be observed after 10 cycles even at a relatively low current density of $1 \mathrm{~mA} \mathrm{~cm}^{-2}$. Such drastically different result further demonstrates the merit of the PI matrix on dendrite suppression.
Moreover, due to the existence of the host matrix, the issue of infinite volume change associated with the 'hostless' Li stripping/plating can now be solved. Even with the complete stripping of $\mathrm{Li}$, the change in electrode thickness was minimal. For example, as shown in Fig. 4f,g, the electrode size was on average $\sim 253 \mu \mathrm{m}$ before stripping and remained at $\sim 247 \mu \mathrm{m}$ after complete stripping, which was merely $\sim 2.4 \%$ of change (a relatively thick electrode was chosen for the more precise determination of thickness variation). However, for bare Li foil, $1 \mathrm{mAh} \mathrm{cm}^{-2}$ capacity represents $\sim 4.85 \mu \mathrm{m}$ thickness of $\mathrm{Li}$ (see Supplementary Method). Therefore, at least tens of microns of electrode thickness fluctuation can be expected for merely a 


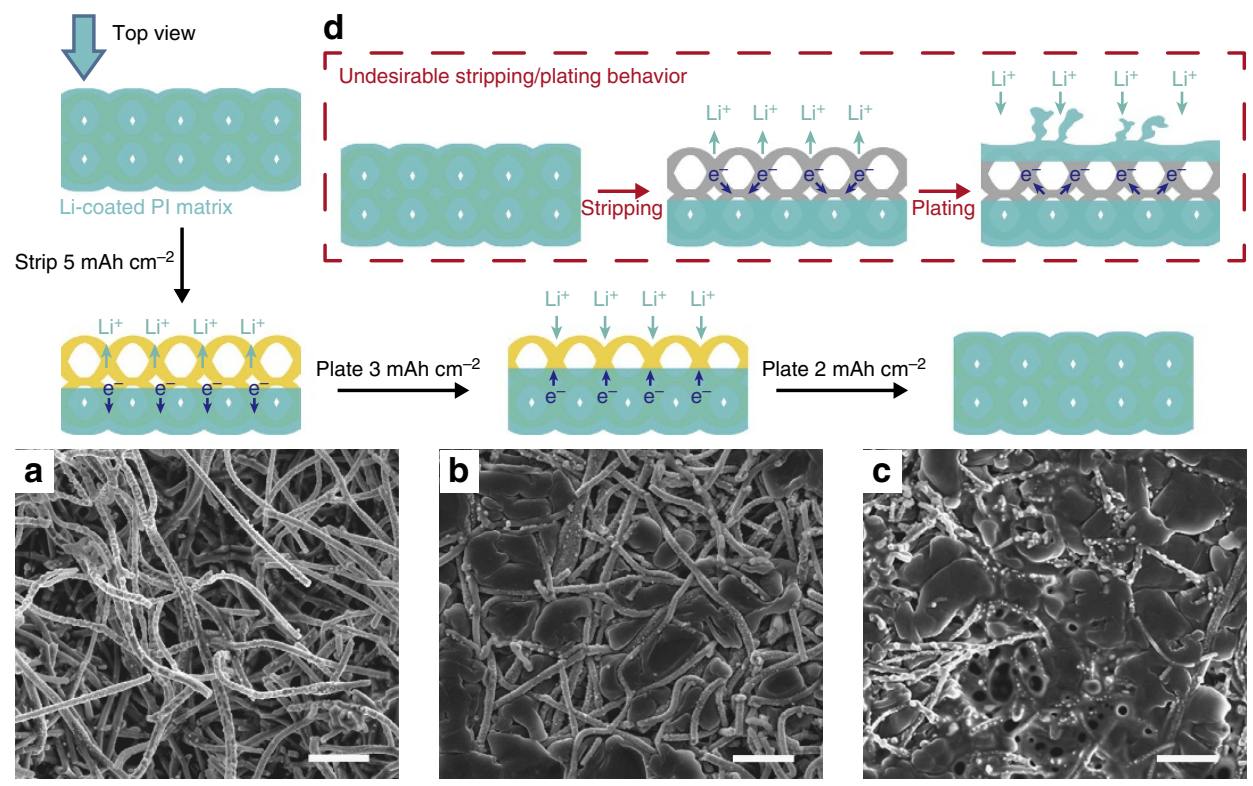

Figure 3 | Well-confined stripping/plating behaviour of the Li-coated PI matrix. Top-view SEM images of (a) the exposed top fibres of the Li-coated PI

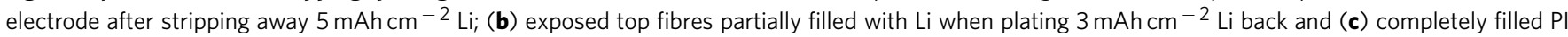
matrix after plating an additional $2 \mathrm{mAh} \mathrm{cm}^{-2} \mathrm{Li}$ back (current density $1 \mathrm{~mA} \mathrm{~cm}^{-2}$, in EC/DEC). The polymeric matrix ensures that $\mathrm{Li}$ is dissolved and deposited from the underlying conductive Li substrate and, as a result, Li is effectively confined into the matrix. (d) Schematic illustrating the alternative undesirable Li stripping/plating behaviour where, after stripping, Li nucleate on the top surface, leading to volume change and dendrites shooting out of the matrix. Scale bars, $5 \mu \mathrm{m}$.
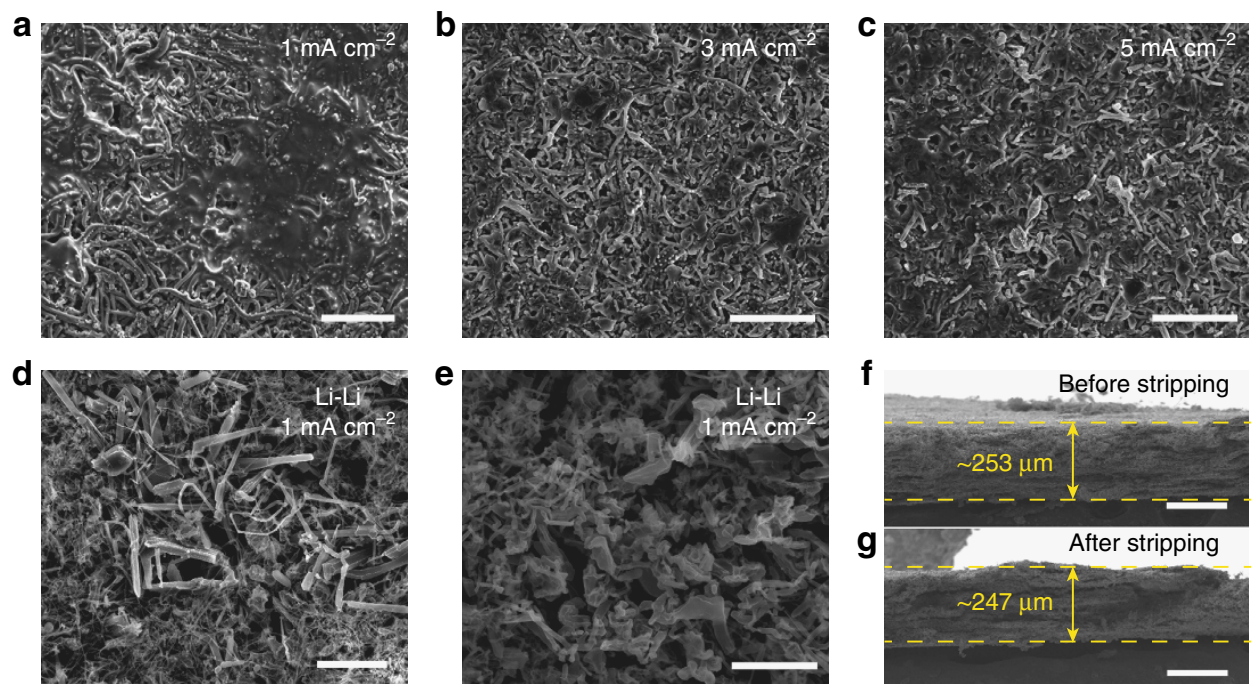

Figure 4 | Morphology of the Li electrodes after cycling at different current densities. Top-view SEM images of the Li-coated PI matrix after 10 cycles of stripping/plating in EC/DEC at a current density of (a) $1 \mathrm{~mA} \mathrm{~cm}^{-2}$, (b) $3 \mathrm{~mA} \mathrm{~cm}^{-2}$ and (c) $5 \mathrm{~mA} \mathrm{~cm}^{-2}$. (d,e) Top-view SEM images of the bare Li electrode after 10 cycles of stripping/plating in EC/DEC at a current density of $1 \mathrm{~mA} \mathrm{~cm}^{-2}$ with large amounts of mossy Li dendrites. Cross-sectional SEM images of the Li-coated PI matrix (f) before and ( $\mathbf{g}$ ) after complete Li stripping, from which no significant volume change can be seen. (Note that the uniform fibrous features in $\mathbf{a}-\mathbf{c}$ are not dendrites but the fibrous matrix, which are distinctly different from the non-uniform, random-sized mossy Li dendrites in d,e). Scale bars, (a-d) $10 \mu \mathrm{m},(\mathbf{e}) 5 \mu \mathrm{m},(\mathbf{f}, \mathbf{g}) 200 \mu \mathrm{m}$.

single layer of Li electrode in a commercial cell. Considering the conventionally applied stacking or rolling battery configuration with multiple layers, the accumulated dimension fluctuation can be tremendous. It is noted that for later cycles, due to the formation of dendritic $\mathrm{Li}$ and thus a porous electrode, the dimension fluctuation can be even larger. Thus, it is apparent that the existence of a stable matrix and the wellconfined $\mathrm{Li}$ cycling behaviour are essential to alleviate the electrode-level volume change, addressing the potential safety concerns.

Electrochemical cycling stability. The galvanostatic cycling performance of the Li-coated PI matrix was studied in both carbonate (EC/DEC) and ether (1 M lithium bis(trifluoromethanesulfonyl)imide in 1:1 w/w 1,3-dioxolane (DOL)/ 

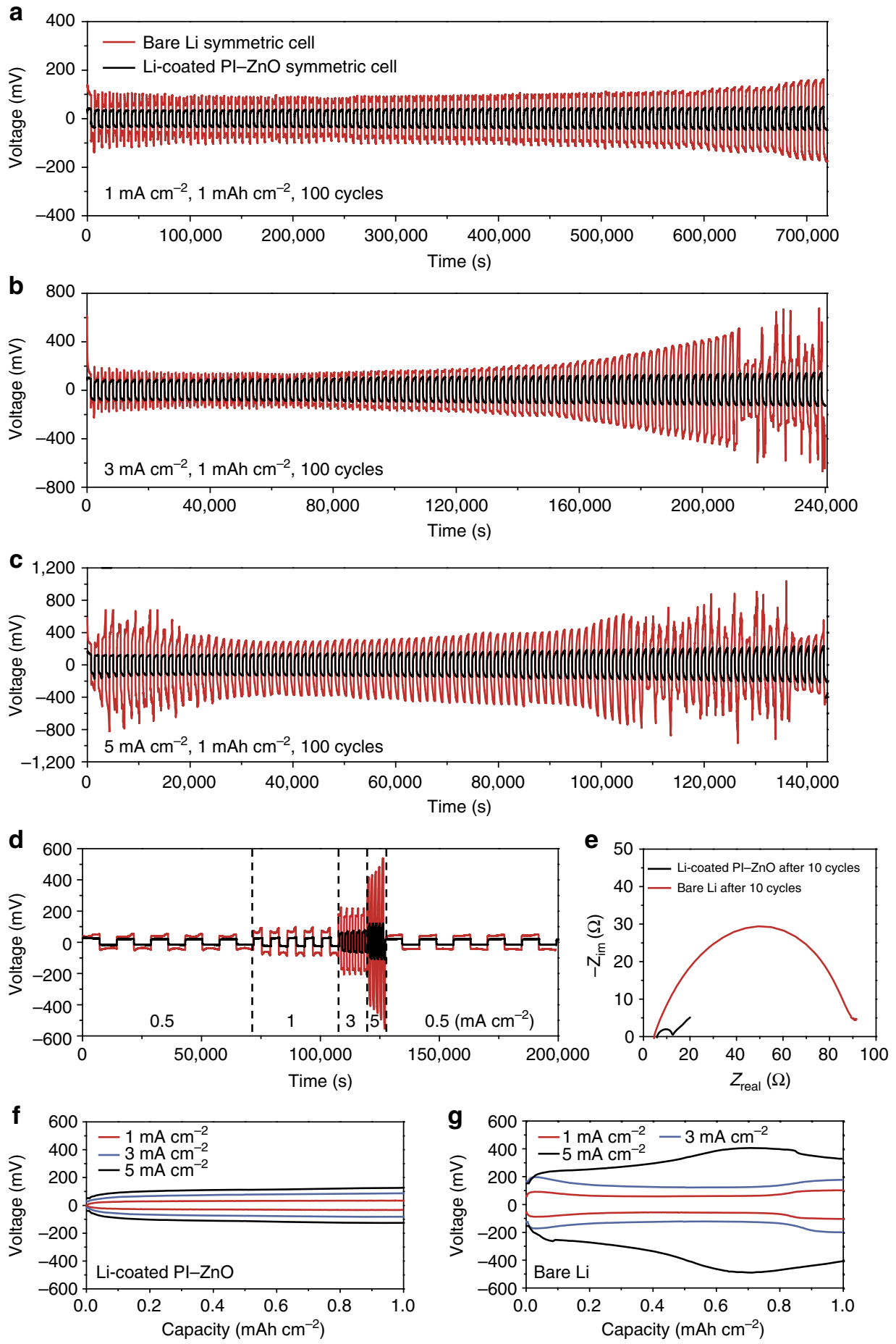

Figure 5 | Electrochemical characterization in EC/DEC electrolyte. Comparison of the cycling stability of the Li-coated PI matrix and the bare Li electrode at a current density of (a) $1 \mathrm{~mA} \mathrm{~cm}^{-2}$, (b) $3 \mathrm{~mA} \mathrm{~cm}^{-2}$ and (c) $5 \mathrm{~mA} \mathrm{~cm}^{-2}$. (d) Rate performance of the Li-coated PI matrix and the bare Li electrode. (e) Nyquist plot of the impedance spectra of the symmetrical Li-coated PI matrix and the bare Li cell after 10 cycles at a current density of $1 \mathrm{~mA} \mathrm{~cm}^{-2}$. Voltage profiles of (f) Li-coated PI matrix and ( $\mathbf{g}$ ) bare Li electrode at different current densities after 10 cycles. The amount of Li cycled was $1 \mathrm{mAh} \mathrm{cm}^{-2}$ in all cases.

1,2-dimethoxyethane (DME) with 1 wt\% lithium nitrate) based electrolyte and compared with bare Li electrode (Fig. 5, Supplementary Figs 12-18). At a current density of $1 \mathrm{~mA} \mathrm{~cm}^{-2}$ in EC/DEC (Fig. 5a), the symmetrical cell of bare Li exhibited a large $\mathrm{Li}$ stripping/plating overpotential $(>100 \mathrm{mV}$ versus $\mathrm{Li}^{+} / \mathrm{Li}$ ), which increased considerably within the first 100 cycles ( $>170 \mathrm{mV}$ in the 100th cycle). In contrast, the Li-coated PI matrix not only showed a much lower overpotential $(\sim 35 \mathrm{mV}$ in the initial cycle) but also achieved very stable cycling for at least 100 cycles $(\sim 40 \mathrm{mV}$ overpotential in the 100th cycle). The difference in cycling stability (Fig. 5b,c) and overpotential (Fig. 5d) became increasingly pronounced at higher current densities. The stripping/plating overpotential for Li-coated PI matrix was $\sim 70$ and $\sim 110 \mathrm{mV}$ at a current densities of 3 and 
$5 \mathrm{~mA} \mathrm{~cm}^{-2}$, respectively, and the values remained constant within 100 cycles. However, the bare Li electrode succumbed to substantial voltage fluctuation at only 88 cycles at a current density of $3 \mathrm{~mA} \mathrm{~cm}^{-2}$ and 75 cycles at a current density of $5 \mathrm{~mA} \mathrm{~cm}^{-2}$, which might be attributed to possible dendriteinduced soft short circuit. Greatly improved cycling stability was also observed as we increased the cycling capacity to $3 \mathrm{mAh} \mathrm{cm}^{-2}$ (Supplementary Fig. 12). Similarly, in DOL/DME electrolyte, the Li-coated PI matrix again outperformed the bare Li electrode (Supplementary Fig. 13). Noticeably, although it is generally recognized that DOL can improve the cycling life of $\mathrm{Li}$ metal anodes due to the formation of a relatively flexible oligomer $\mathrm{SEI}^{44}$, the bare $\mathrm{Li}$ electrode still exhibited a necking behaviour (the overpotential first decreases, then increases) during cycling, which is a characteristic sign for dendrite formation in early stage and SEI accumulation later ${ }^{37}$. Nevertheless, the Li-coated PI matrix maintained flat, constant cycling profiles and reduced overpotential at all current densities. Such exceptional long-term cycling performance is a good indicator of the superior $\mathrm{CE}$ and more uniform $\mathrm{Li}$ deposition/dissolution of the Li-coated PI electrode. Finally, since it is technically challenging to directly determine the $\mathrm{CE}$ of electrodes with pre-stored $\mathrm{Li}$ through $\mathrm{Li}$ plating/stripping as in previous studies ${ }^{27,34}$, we paired our electrode with high-areal-capacity $\mathrm{Li}_{4} \mathrm{Ti}_{5} \mathrm{O}_{12}$ (LTO, $\sim 3 \mathrm{mAh} \mathrm{cm}^{-2}$ ) electrode as an indirect method to study the CE of the electrode (Supplementary Fig. 14). Li-coated PI electrode $\left(\sim 10 \mathrm{mAh} \mathrm{cm}^{-2}\right)$ exhibited quite stable cycling comparable to the highly oversized $\mathrm{Li}$ metal counterpart $(750 \mu \mathrm{m} \mathrm{Li})$, while bare $50 \mu \mathrm{m} \mathrm{Li}$ foil $\left(\sim 10 \mathrm{mAh} \mathrm{cm}^{-2}\right)$ started to decay at $\sim 20$ cycles. The results gave rise to semiquantitative $\mathrm{CE}$ values and illustrate the superior CE of the Li-coated PI electrode (see Supplementary Note 1).

\section{Discussion}

Two key factors contributed to the excellent electrochemical performance of the Li-coated PI matrix, namely, the porous nature of the electrode and the non-conducting nature of the exposed matrix surface after $\mathrm{Li}$ stripping. As mentioned previously, molten Li was drawn into the matrix preferentially along the fibres during the $\mathrm{Li}$ coating step, resulting in a porous $\mathrm{Li}$ electrode. The high porosity can be further confirmed by the fast electrolyte uptake during cell assembly (Supplementary Fig. 15). Such high porosity increased the surface area of the electrode, which can in turn significantly reduce the effective current density during cycling. As a result, the Li stripping/plating overpotential was much smaller for the Li-coated PI matrix, especially at high current densities (Fig. 5d). Correspondingly, the electrochemical impedance spectroscopy revealed a much reduced interfacial charge transfer resistance for the Li-coated PI matrix compared to bare $\mathrm{Li}(\sim 10$ times lower in EC/DEC, Fig. 5e and Supplementary Fig. 16). More importantly, the reduced effective current density rendered the Li-coated PI matrix a flat stripping/plating voltage profile (Fig. 5f,g and Supplementary Fig. 17). For the bare Li electrode, large 'overpotential bumps' at the beginning and the end of each stripping or plating process can be observed, especially for the early cycles (Supplementary Fig. 18). This phenomenon can be explained by the high specific kinetic hindrance for non-uniform Li dissolution/deposition at high current density, which has been investigated in great detail in previous reports ${ }^{37,45}$. The fluctuation attenuated in later cycles due to the formation of mossy $\mathrm{Li}$, which increased the surface area of the electrode, reducing the effective current density. However, when compared to the fluctuated profile of the bare Li electrode, the voltage profile of Li-coated PI was flat at all current densities throughout the cycling, clearly demonstrating the advantages of the porous electrode. In addition, it is well known that the effective current density during Li stripping/plating has a crucial impact on the dendrite formation and growth ${ }^{37,46}$. Lower effective current density results in reduced electrolyte decomposition and related SEI formation during cycling so as to suppress the dendrite growth ${ }^{37}$. Therefore, the porous electrode structure, in addition to the non-conducting polymeric matrix, which led to the well-confined $\mathrm{Li}$ dissolution/deposition behaviour, effectively ensured the dendrite-free cycling of the Li-coated PI electrode, giving rise to stable long-term performance.

In conclusion, our work demonstrates the method of obtaining a free-standing, porous metallic $\mathrm{Li}$ anode by infusing molten $\mathrm{Li}$ into a core-shell PI-ZnO matrix. The excellent heat resistance and chemical stability of the PI fibres guaranteed the structural integrity of the matrix during $\mathrm{Li}$ coating and the subsequent battery cycling, while the conformal ALD $\mathrm{ZnO}$ coating provided the driving force for the molten Li infusion. Noticeably, the exposed non-conducting fibres after Li stripping prevented the direct plating of $\mathrm{Li}$ on the top surface of the electrode, which effectively confined $\mathrm{Li}$ within the matrix. In this manner, dendrite-free and minimum-volume-change Li stripping/plating can be successfully achieved, addressing the biggest concerns regarding Li metal anode. Remarkably, different from the dense bare Li electrode, the large porosity of the Li-coated PI electrode considerably decreased the effective current density during cycling. As a result, flat voltage profiles and long-term cycling stability can be realized even at a high current density of $5 \mathrm{~mA} \mathrm{~cm}^{-2}$. The benefits of a non-conducting polymeric matrix and electrode porosity shed new light on the design principles of metallic $\mathrm{Li}$ anodes and open up new opportunities to the realization of the next-generation high-energy-density battery systems based on Li metal chemistries.

\section{Methods}

Electrospinning of the PI matrix. $15 \mathrm{wt} \%$ PI powder (DuPont CP-0650) was dissolved in $\mathrm{N}$-methyl-2-pyrrolidone and stirred at 750 r.p.m. in a $60^{\circ} \mathrm{C}$ oil bath overnight to afford a homogeneous solution. Subsequently, the solution was loaded into a glass syringe with a stainless steel needle tip, which is connected to a voltage supply (ES30P-5 W, Gamma High Voltage Research). The applied potential on the needle was $15 \mathrm{kV}$, the distance between the needle tip and the graphite paper collector was $15 \mathrm{~cm}$ and the pumping rate was $10 \mu \mathrm{min}^{-1}$. In addition, a negative voltage of $1 \mathrm{kV}$ was applied at the collector to improve the homogeneity of the electrospun film. The thickness of the electrospun PI matrix can be tuned easily by adjusting the electrospinning time.

Atomic layer deposition. The conformal $\mathrm{ZnO}$ layer was coated on the PI fibres via ALD using the Cambridge Nanotech Savannah $\mathrm{S} 100$ at $80^{\circ} \mathrm{C}$ with diethyl zinc (DEZn) and DI water as precursors. The pulse times for DEZn and DI water were $15 \mathrm{~ms}$ each, with $60 \mathrm{~s}$ waiting between each pulse. Approximately 300 cycles were needed to obtain a $\mathrm{ZnO}$ coating with desirable Li wetting property.

Li coating of the core-shell PI-ZnO matrix. The Li coating process was carried out in an argon-filled glovebox with sub-p.p.m. $\mathrm{O}_{2}$ level. In a typical process, freshly scraped Li foil (99.9\%, Alfa Aesar) was put into a stainless-steel crucible and heated to melt on a hotplate (VWR). Subsequently, the edge of the core-shell $\mathrm{PI}-\mathrm{ZnO}$ matrix (punched into $1-\mathrm{cm}^{2}$ discs) was put into contact with the molten Li. Li can steadily climb up and wet the whole matrix, affording the final Li electrode.

Electrochemical measurements. The processes of Li stripping and plating were studied using a symmetric cell configuration by assembling the electrodes into 2,032-type coin cells. The electrolytes employed were either $1 \mathrm{M}$ lithium hexafluorophosphate $\left(\mathrm{LiPF}_{6}\right.$ ) in 1:1 EC/DEC (BASF Selectilyte LP40) or $1 \mathrm{M}$ lithium bis(trifluoromethanesulfonyl)imide in 1:1 w/w DOL/DME with $1 \mathrm{wt} \%$ lithium nitrate additive. The separator used was Celgard 2325 ( $25 \mu \mathrm{m} \mathrm{PP/PE} / \mathrm{PP})$. The control bare Li cells were assembled using freshly scraped Li foil. Galvanostatic cycling was conducted on a standard eight-channel battery tester (Wuhan LAND Electronics Co., Ltd.). A constant current was applied to the electrodes during repeated stripping/plating while the potential was recorded over time. The impedance measurements were carried out using a Biologic VMP3 multichannel system. For the half-cell study, the LTO electrodes were prepared by mixing LTO (MTI), 
polyvinylidene fluoride (MTI) and carbon black (TIMCAL) in the ratio of 8:1:1 with $\mathrm{N}$-methyl-2-pyrrolidone as the solvent. The areal mass loading of the LTO electrodes was $\sim 20 \mathrm{mg} \mathrm{cm}^{-2}$.

Characterization. SEM images were taken using a FEI XL30 Sirion scanning electron microscope at an acceleration voltage of $5 \mathrm{kV}$. In order to observe the surface morphology of Li after cycling, the electrodes were disassembled from the coil cell in the glovebox followed by gentle rinse in DOL. Scanning transmission electron microscopy images, the corresponding energy-dispersive $\mathrm{X}$-ray elemental mapping and line scan were obtained on a FEI Tecnai G2 F20 X-TWIN. XRD patterns were recorded on a PANalytical X'Pert instrument. Noticeably, the Li electrode was loaded on a glass slide and covered with Kapton tape during XRD measurements to avoid direct contact with air. Fourier transform infrared spectra were recorded on a Nicolet iS50 FT/IR Spectrometer (Thermo Scientific). Thermogravimetric analysis was performed on a TA Instrument Q500 TGA in air at a heating rate of $5^{\circ} \mathrm{Cmin}^{-1}$. $\mathrm{N}_{2}$ sorption studies were performed using a Micromeritics ASAP 2,020 adsorption apparatus at $77 \mathrm{~K}$ and at pressure up to 1 bar after the samples were first degassed at $180^{\circ} \mathrm{C}$ overnight. The Brunauer-Emmett-Teller surface area was calculated using the adsorption data in a relative pressure ranging from 0.1 to 0.3 .

\section{References}

1. Armand, M. \& Tarascon, J. M. Building better batteries. Nature 451, 652-657 (2008).

2. Chan, C. K. et al. High-performance lithium battery anodes using silicon nanowires. Nat. Nanotechnol. 3, 31-35 (2008).

3. Bruce, P. G., Freunberger, S. A., Hardwick, L. J. \& Tarascon, J. M. Li- $\mathrm{O}_{2}$ and Li-S batteries with high energy storage. Nat. Mater. 11, 19-29 (2012).

4. Xu, W. et al. Lithium metal anodes for rechargeable batteries. Energy Environ. Sci. 7, 513-537 (2014).

5. Kim, H. et al. Metallic anodes for next generation secondary batteries. Chem. Soc. Rev. 42, 9011-9034 (2013).

6. Aurbach, D., Zinigrad, E., Cohen, Y. \& Teller, H. A short review of failure mechanisms of lithium metal and lithiated graphite anodes in liquid electrolyte solutions. Solid State Ion. 148, 405-416 (2002).

7. Brissot, C., Rosso, M., Chazalviel, J. N. \& Lascaud, S. Dendritic growth mechanisms in lithium/polymer cells. J. Power Sources 81, 925-929 (1999).

8. Peled, E. The electrochemical-behavior of alkali and alkaline-earth metals in non-aqueous battery systems-the solid electrolyte interphase model. J. Electrochem. Soc. 126, 2047-2051 (1979).

9. Cohen, Y. S., Cohen, Y. \& Aurbach, D. Micromorphological studies of lithium electrodes in alkyl carbonate solutions using in situ atomic force microscopy. J. Phys. Chem. B 104, 12282-12291 (2000).

10. Aurbach, D., Gofer, Y. \& Langzam, J. The correlation between surfacechemistry, surface-morphology, and cycling efficiency of lithium electrodes in a few polar aprotic systems. J. Electrochem. Soc. 136, 3198-3205 (1989).

11. Gireaud, L., Grugeon, S., Laruelle, S., Yrieix, B. \& Tarascon, J. M. Lithium metal stripping/plating mechanisms studies: a metallurgical approach. Electrochem. Commun. 8, 1639-1649 (2006).

12. Bhattacharyya, R. et al. In situ NMR observation of the formation of metallic lithium microstructures in lithium batteries. Nat. Mater. 9, 504-510 (2010).

13. Harry, K. J., Hallinan, D. T., Parkinson, D. Y., MacDowell, A. A. \& Balsara, N. P. Detection of subsurface structures underneath dendrites formed on cycled lithium metal electrodes. Nat. Mater. 13, 69-73 (2014).

14. Chandrashekar, S. et al. Li-7 MRI of Li batteries reveals location of microstructural lithium. Nat. Mater. 11, 311-315 (2012).

15. Kamaya, N. et al. A lithium superionic conductor. Nat. Mater. 10, 682-686 (2011).

16. Murugan, R., Thangadurai, V. \& Weppner, W. Fast lithium ion conduction in garnet-type $\mathrm{Li}_{7} \mathrm{La}_{3} \mathrm{Zr}_{2} \mathrm{O}_{12}$. Angew. Chem. Int. Ed. 46, 7778-7781 (2007).

17. Li, J. C., Ma, C., Chi, M. F., Liang, C. D. \& Dudney, N. J. Solid electrolyte: the key for high-voltage lithium batteries. Adv. Energy Mater. 5 doi:10.1002/ aenm.201401408 (2015).

18. Croce, F., Persi, L., Ronci, F. \& Scrosati, B. Nanocomposite polymer electrolytes and their impact on the lithium battery technology. Solid State Ion. 135, 47-52 (2000).

19. Bouchet, R. et al. Single-ion BAB triblock copolymers as highly efficient electrolytes for lithium-metal batteries. Nat. Mater. 12, 452-457 (2013).

20. Kim, K. H. et al. Characterization of the interface between $\mathrm{LiCoO}_{2}$ and $\mathrm{Li}_{7} \mathrm{La}_{3} \mathrm{Zr}_{2} \mathrm{O}_{12}$ in an all-solid-state rechargeable lithium battery. J. Power Sources 196, 764-767 (2011).

21. Zhang, T. et al. A novel high energy density rechargeable lithium/air battery. Chem. Commun. 46, 1661-1663 (2010).

22. $\mathrm{Xu}, \mathrm{K}$. Electrolytes and interphases in Li-ion batteries and beyond. Chem. Rev. 114, 11503-11618 (2014).
23. Li, W. et al. The synergetic effect of lithium polysulfide and lithium nitrate to prevent lithium dendrite growth. Nat. Commun. 6, 7436 (2015).

24. Lu, Y. Y., Tu, Z. Y. \& Archer, L. A. Stable lithium electrodeposition in liquid and nanoporous solid electrolytes. Nat. Mater. 13, 961-969 (2014).

25. Qian, J. et al. High rate and stable cycling of lithium metal anode. Nat. Commun. 6, 6362 (2015).

26. Suo, L. M., Hu, Y. S., Li, H., Armand, M. \& Chen, L. Q. A new class of solventin-salt electrolyte for high-energy rechargeable metallic lithium batteries. Nat. Commun. 4, 1481 (2013).

27. Ding, F. et al. Dendrite-free lithium deposition via self-healing electrostatic shield mechanism. J. Am. Chem. Soc. 135, 4450-4456 (2013).

28. Umeda, G. A. et al. Protection of lithium metal surfaces using tetraethoxysilane. J. Mater. Chem. 21, 1593-1599 (2011).

29. Ishikawa, M., Morita, M. \& Matsuda, Y. In situ scanning vibrating electrode technique for lithium metal anodes. J. Power Sources 68, 501-505 (1997).

30. Kang, I. S., Lee, Y. S. \& Kim, D. W. Improved cycling stability of lithium electrodes in rechargeable lithium batteries. J. Electrochem. Soc. 161, A53-A57 (2014).

31. Ji, X. L. et al. Spatially heterogeneous carbon-fiber papers as surface dendritefree current collectors for lithium deposition. Nano Today 7, 10-20 (2012).

32. Ryou, M. H. et al. Excellent cycle life of lithium-metal anodes in lithium-ion batteries with mussel-inspired polydopamine-coated separators. Adv. Energy Mater. 2, 645-650 (2012).

33. Kozen, A. C. et al. Next-generation lithium metal anode engineering via atomic layer deposition. ACS Nano 9, 5884-5892 (2015).

34. Zheng, G. Y. et al. Interconnected hollow carbon nanospheres for stable lithium metal anodes. Nat. Nanotechnol. 9, 618-623 (2014).

35. Yan, K. et al. Ultrathin two-dimensional atomic crystals as stable interfacial layer for improvement of lithium metal anode. Nano Lett. 14, 6016-6022 (2014).

36. Liang, Z. et al. Polymer nanofiber-guided uniform lithium deposition for battery electrodes. Nano Lett. 15, 2910-2916 (2015).

37. Heine, J. et al. Coated lithium powder (CLiP) electrodes for lithium-metal batteries. Adv. Energy Mater. 4 doi:10.1002/aenm.201300815 (2014).

38. Yang, C.-P., Yin, Y.-X., Zhang, S.-F., Li, N.-W. \& Guo, Y.-G. Accommodating lithium into 3D current collectors with a submicron skeleton towards long-life lithium metal anodes. Nat. Commun. 6, 8058 (2015).

39. Wright, W. W. \& Hallden-Abberton, M. in Ullmann's Encyclopedia of Industrial Chemistry (Wiley-VCH Verlag GmbH \& Co. KGaA, 2000).

40. Fu, Z. W., Huang, F., Zhang, Y., Chu, Y. \& Qin, Q. Z. The electrochemical reaction of zinc oxide thin films with lithium. J. Electrochem. Soc. 150, A714-A720 (2003).

41. Taberna, L., Mitra, S., Poizot, P., Simon, P. \& Tarascon, J. M. High rate capabilities $\mathrm{Fe}_{3} \mathrm{O}_{4}$-based $\mathrm{Cu}$ nano-architectured electrodes for lithium-ion battery applications. Nat. Mater. 5, 567-573 (2006).

42. Grugeon, S. et al. Particle size effects on the electrochemical performance of copper oxides toward lithium. J. Electrochem. Soc. 148, A285-A292 (2001).

43. Mrsevic, M., Dusselberg, D. \& Staudt, C. Synthesis and characterization of a novel carboxyl group containing (co)polyimide with sulfur in the polymer backbone. Beilstein J. Org. Chem. 8, 776-786 (2012).

44. Aurbach, D., Youngman, O., Gofer, Y. \& Meitav, A. The electrochemical behaviour of 1,3-dioxolane- $\mathrm{LiClO}_{4}$ solutions-I. Uncontaminated solutions. Electrochim. Acta 35, 625-638 (1990).

45. Bieker, G., Winter, M. \& Bieker, P. Electrochemical in situ investigations of SEI and dendrite formation on the lithium metal anode. Phys. Chem. Chem. Phys. 17, 8670-8679 (2015).

46. Kim, J. S., Baek, S. H. \& Yoon, W. Y. Electrochemical behavior of compacted lithium powder electrode in $\mathrm{Li} / \mathrm{V}_{2} \mathrm{O}_{5}$ rechargeable battery. J. Electrochem. Soc 157, A984-A987 (2010).

\section{Acknowledgements}

Y.C. acknowledges the support from the Assistant Secretary for Energy Efficiency and Renewable Energy, Office of Vehicle Technologies of the US Department of Energy under the Battery Materials Research (BMR) program.

\section{Author contributions}

Y.L., D.L. and Y.C. conceived the idea. Y.L. and D.L. carried out the materials synthesis and electrochemical tests. J.Z. carried out the TEM characterization. K.Y. and Z.L. provided important experimental insights. Y.L., D.L. and Y.C. co-wrote the paper. All the authors discussed the results and commented on the manuscript.

\section{Additional information}

Supplementary Information accompanies this paper at http://www.nature.com/ naturecommunications

Competing financial interests: The authors declare no competing financial interests. 
Reprints and permission information is available online at http://npg.nature.com/ reprintsandpermissions/

How to cite this article: Liu, Y. et al. Lithium-coated polymeric matrix as a minimum volume-change and dendrite-free lithium metal anode. Nat. Commun. 7:10992 doi: 10.1038/ncomms10992 (2016). (c) (i) This work is licensed under a Creative Commons Attribution 4.0 International License. The images or other third party material in this article are included in the article's Creative Commons license, unless indicated otherwise in the credit line; if the material is not included under the Creative Commons license, users will need to obtain permission from the license holder to reproduce the material. To view a copy of this license, visit http://creativecommons.org/licenses/by/4.0/ 